\title{
Experiments in a rotating Hele-Shaw cell
}

\author{
Ll. Carrillo, F. X. Magdaleno, J. Casademunt, and J. Ortín \\ Departament d'Estructura i Constituents de la Matèria, Facultat de Física, Universitat de Barcelona, \\ Diagonal 647, E-08028 Barcelona, Spain
}

(Received 23 April 1996)

\begin{abstract}
We introduce a modification to Hele-Shaw flows consisting of a rotating cell. A viscous fluid (oil) is injected at the rotation axis of the cell, which is open to air. The morphological instability of the oil-air interface is thus driven by centrifugal force and is controlled by the density (not viscosity) difference. We derive the linear dispersion relation and verify the maximum growth rate selection of initial patterns within experimental uncertainty. The nonlinear growth regime is studied in the case of vanishing injection rate. Several characteristic lengths are studied to quantify the patterns obtained. Experimental data exhibit good collapse for two characteristic lengths, namely, the radius of gyration and the radial finger length, which in the nonlinear regime appear to grow linearly in time. [S1063-651X(96)02511-1]
\end{abstract}

PACS number(s): 47.20.Ma, 47.20.Hw, 47.55.Kf, 68.10.-m

\section{INTRODUCTION}

The study of the morphological instability of an interface between two immiscible fluids confined in a Hele-Shaw cell has received a great deal of attention in the past decade as a prototypical system to study generic features of interfacial pattern formation in nonequilibrium systems [1]. This fluid system has in fact important aspects in common with other more complicated systems from different contexts including flow in porous media, crystal growth, chemical electrodeposition, flame propagation, etc. [2]. The study of viscous fingering in Hele-Shaw cells has the great advantage of being the simplest model from both theoretical and experimental points of view. Studies of Hele-Shaw two-fluid flows can be grouped into two categories according to the two basic geometries, namely, the linear or channel geometry and the radial or circular one. In the former the focus has been on the study of the mechanisms of steady-state selection [3], whereas the latter has focused on the asymptotic morphologies in connection with the concepts of fractal growth [4].

The success of the studies of viscous fingering as a prototypical system for interfacial pattern formation has lead more recently to the introduction of perturbations or modifications of the problem in order to enrich it in a controlled way and thus obtain insight into more complicated features of related problems. A recent review of contributions along these lines can be found in Ref. [5] and references therein.

In the same spirit we introduce here a controlled modification on the radial viscous fingering setup, consisting of a rotating horizontal Hele-Shaw cell. To some extent this problem can be seen as the counterpart of the gravity-driven experiment in rectangular Hele-Shaw cells, but now in the circular geometry. As opposed to the classical case, here the injected fluid will typically be more dense, for instance, oil displacing air, in order to obtain a morphological instability of the interface. It can be argued that Coriolis forces are negligible within the framework in which the Hele-Shaw approximation is valid. If they become noticeable at some point, they would manifest by breaking the radial symmetry of the problem.

One of the main motivations for this study comes from the observation of interesting dynamical phenomena for low viscosity contrast in gravity-driven experiments [6]. In those cases, the morphological instability is basically originated by the density difference and not by the viscosity difference of the two fluids. This allows one to explore the region of low viscosity contrast. Simulations [7], experiments [6], and later theoretical analysis [8] showed that the viscosity contrast played a crucial role in the deeply nonlinear regime, with important consequences, for instance, on the selection of the single-finger steady state [8]. Since the screening mechanisms of finger competition and the resulting interface morphologies depended strongly on viscosity contrast, the question arises as to whether such a parameter plays a crucial role in the radial nonlinear growth case too.

The relation between injection and centrifugal driving in the circular geometry introduces some important differences with respect to the correspondence of injection vs gravity driving in the channel geometry. In the latter case, an exact parameter mapping exists between the two cases, which become equivalent in the appropriate dimensionless formulation together with a change of frame of reference. In the circular geometry this mapping exists only in the linear regime (small amplitude perturbations of a circular interface) since the advance of the interface cannot be absorbed in a change of reference frame. The closest analog to a gravity driven experiment would be the case of vanishing injection rate, in which the total mass of the inner fluid is conserved. Even in this case there is an important difference with the gravity driven counterpart in that the centrifugal force depends on radial distance, while gravity is constant. This not only modifies the balance between stabilizing and destabilizing effects of viscosity and density contrasts, but may lead to new morphologies in the highly nonlinear regime. An additional motivation for this experiment is the study of the existence of topological singularities in Hele-Shaw flows, such as the breakup of the interface into bubbles. Such phenomena have received much attention from a theoretical point of view in different circumstances [9] and were significantly present in experiments of low contrast gravity-driven experiments. In our case they could be potentially more important due to the growth of the driving force with distance. We will 
not address this point in the present paper and will focus, as a first step, on the formulation of the problem and the characterization of its basic features in the limit of high viscosity contrast, deferring a more detailed morphological and dynamical study to future work.

Finally, one of the interesting features of the centrifugal driving of the instability is that it allows one to study the case of vanishing injection rate. This considerably simplifies the problem, and data collapse to simple scaling laws seems more feasible, as suggested by existing results on the circular geometry [4] and particularly by related experiments such as in Ref. [10]. In the present paper the study of nonlinear growth will be restricted to the case of vanishing injection rate.

The rest of the paper is organized as follows. Section II is devoted to the formulation of the problem and discussion of the linear instability. In Sec. III we describe the experimental setup and in Sec. IV we present the experimental results. Some final considerations are left for Sec. V.

\section{FORMULATION AND LINEAR STABILITY ANALYSIS}

Consider two immiscible fluids of viscosities $\mu_{1}, \mu_{2}$ and densities $\rho_{1}, \rho_{2}$, between the two glass plates of a Hele-Shaw cell of gap spacing $b$. Within the usual high friction approximation, when the cell rotates with an angular velocity $\Omega$, Coriolis forces can be neglected and the flow is potential, that is, $\mathbf{v}=\boldsymbol{\nabla} \phi$, where the velocity potential $\phi$ is defined for each fluid by

$$
\phi_{i}=-M_{i}\left(p_{i}-\frac{1}{2} \rho_{i} \Omega^{2} r^{2}\right)
$$

and the mobility $M_{i}$ is given by $M_{i}=b^{2} / 12 \mu_{i}$. The index $i$ is 1 for the inner fluid and 2 for the outer one. With the incompressibility condition, the problem is then completely specified, in its simplest form, by the bulk equation

$$
\nabla^{2} \phi_{i}=0
$$

and the two boundary conditions at the interface

$$
\begin{gathered}
p_{1}-p_{2}=\sigma \kappa, \\
v_{n}=\mathbf{n} \cdot \nabla \phi_{1}=\mathbf{n} \cdot \nabla \phi_{2} .
\end{gathered}
$$

Equation (3) is the usual Laplace pressure jump at the interface, where $\sigma$ is surface tension. The sign convention for the curvature $\kappa$ is such that a circular interface has positive curvature. Equation (4) is the continuity condition, with the unit normal $\mathbf{n}$ directed from fluid 1 to 2 .

For the case of a circular interface of radius $R$, the velocity potential takes the form

$$
\phi_{i}^{(0)}=\frac{Q}{2 \pi} \ln \left(\frac{r}{R}\right)+\phi_{i 0},
$$

where $Q$ is the aerial injection rate (area covered per unit time). If we now assume an infinitesimal perturbation of the circular interface of the form $a=A \delta(t) e^{i n \theta}$ we can derive the corresponding linear dispersion relation $\omega(n)$ defined by

$$
\frac{\dot{\delta}}{\delta}=\omega(n)+\mathcal{O}(\delta),
$$

following the same steps as in Ref. [11]. In the Appendix we give an alternative formulation of the problem based on conformal mapping techniques and outline the derivation of the linear dispersion relation within that framework. With an ansatz of the form

$$
\begin{aligned}
& \phi_{1}=\phi_{1}^{(0)}+\beta\left(\frac{r}{R}\right)^{n} e^{i n \theta}, \\
& \phi_{2}=\phi_{2}^{(0)}-\beta\left(\frac{R}{r}\right)^{n} e^{i n \theta},
\end{aligned}
$$

the continuity condition Eq. (4) determines $\beta$ in terms of the rest of parameters as

$$
\beta=\frac{A \delta}{n}\left[\frac{Q}{2 \pi R}+R \frac{\dot{\delta}}{\delta}\right] .
$$

Using the pressure drop condition Eq. (3), we find

$$
\omega(n)=-\frac{Q}{2 \pi R^{2}}+\widetilde{\Omega} n-\frac{\widetilde{\sigma}}{R^{3}} n\left(n^{2}-1\right),
$$

where

$$
\widetilde{\Omega}=\frac{\Omega^{2} b^{2}}{12} \frac{\rho_{1}-\rho_{2}}{\mu_{1}+\mu_{2}}-\frac{Q}{2 \pi R^{2}} \frac{\mu_{1}-\mu_{2}}{\mu_{1}+\mu_{2}}
$$

and

$$
\widetilde{\sigma}=\frac{b^{2}}{12} \frac{\sigma}{\mu_{2}+\mu_{1}} .
$$

Note that, in general, both $Q$ and $R$ are time dependent, so the actual relaxation or growth of the modes is not exponential. In particular, for a circular interface we trivially have $Q /\left(2 \pi R^{2}\right)=\dot{R} / R$. We will be mostly concerned with the case of positive, constant $Q$, in which case $R \sim t^{1 / 2}$. If the experiment is performed at constant injection pressure, then we have $R^{2} \ln R \sim t$. Equation (10) reproduces the usual result for a circular geometry when $\Omega=0$ and reduces to the case of a planar interface in a channel geometry $\omega(q)=q\left[U_{\infty}\left(\mu_{2}-\mu_{1}\right) /\left(\mu_{2}+\mu_{1}\right)-\widetilde{\sigma} q^{2}\right]$, in the limits $R \rightarrow \infty$ and $|Q| \rightarrow \infty$ with $|Q| / 2 \pi R=U_{\infty}$ and $n / R=q$.

The different feature of Eq. (10) is the presence of the first term on the right-hand side of Eq. (11), which is proportional to the density difference. The instability will be governed by the sign and size of $\widetilde{\Omega}$. For positive $Q$, it is clear that for a typical situation in which the driving fluid is both more viscous and more dense, such as for oil displacing air, the effects of the viscosity difference and density difference are opposite. The former is stabilizing and the latter distabilizing. Moreover, only the stabilizing effect of viscosity depends on the radius $R$, so the balance of the two terms will favor the instability for large $R$ or equivalently long times. Finally, the signs of the two terms can be changed separately so the problem is considerably enriched when an angular velocity $\Omega$ is introduced, already at the level of the linear 


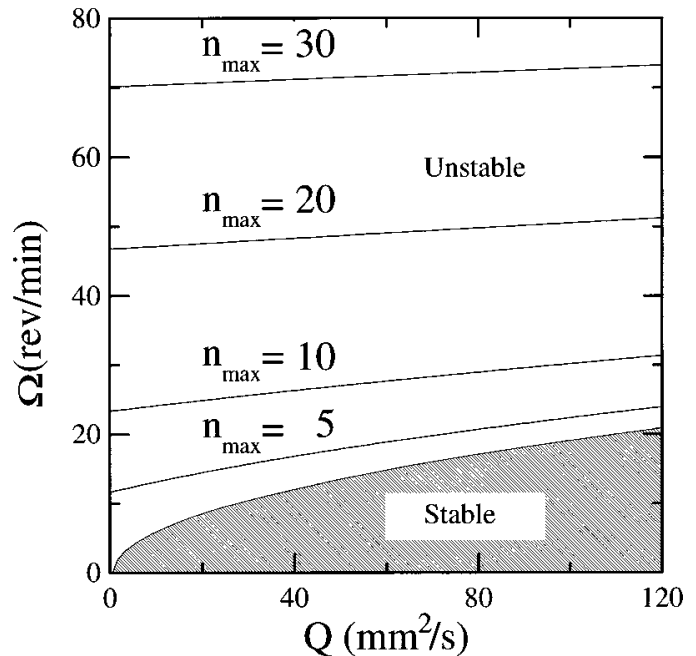

FIG. 1. Prediction of a linear stability analysis for $n_{\max }$, the most unstable mode, as a function of two control parameters $\Omega$ (angular velocity of the cell) and $Q$ (aerial injection rate). The other parameters are $b=0.5 \mathrm{~mm}, R_{0}=100 \mathrm{~mm}, \sigma=20.7 \mathrm{mN} / \mathrm{m}, \nu=50$ $\mathrm{mm}^{2} / \mathrm{s}$, and $\rho=1000 \mathrm{~kg} / \mathrm{m}^{3}$.

instability. Notice that $\Omega$ appears squared, since the effect of the rotation cannot depend on its sign, so the dependence on this control parameter is rather strong.

The most important aspect of Eqs. (10) and (11), however, is the fact that the interface may be unstable regardless of the sign of the viscosity difference, and the nonlinear regime can now be studied in terms of viscosity contrast, including the region of low viscosity contrast. For the sake of simplicity, we will restrict ourselves mostly to $Q=0$, in which case the viscosity contrast is manifestly irrelevant at the linear level. The absence of this extra parameter makes the collapse of the experimental data much simpler, as we will see in the following sections.

From Eq. (10) we can also obtain some direct consequences on the $n$ dependence of the linear dispersion relation. The zero mode that corresponds to a uniform expansion of the circle behaves as in the classical case. It decays (grows) for positive (negative) $Q$ as a consequence of mass conservation and is marginal for $Q=0$. The mode $n=1$ corresponds to a global off-center shift of the circular interface. The stability of this mode is given solely by the sign of $\widetilde{\Omega}$ and is independent of surface tension since it preserves the circular shape. For $n>2$ the stability depends on the interplay of the two terms of Eq. (10). As usual, large $n$ is dominated by the stabilizing effect of surface tension, resulting, in the unstable case, in a finite band of unstable modes. For positive $\widetilde{\Omega}$, the most unstable mode is given by the closest integer to the maximum of Eq. (10) with respect to $n$, which reads

$$
n_{\max }=\sqrt{\frac{1}{3}\left(1+\frac{\widetilde{\Omega} R^{3}}{\widetilde{\sigma}}\right)} .
$$

Equation (13) is illustrated in Fig. 1.

Finally, for the case $Q=0$, the marginal mode $\omega\left(n_{c}\right)=0$ is given by

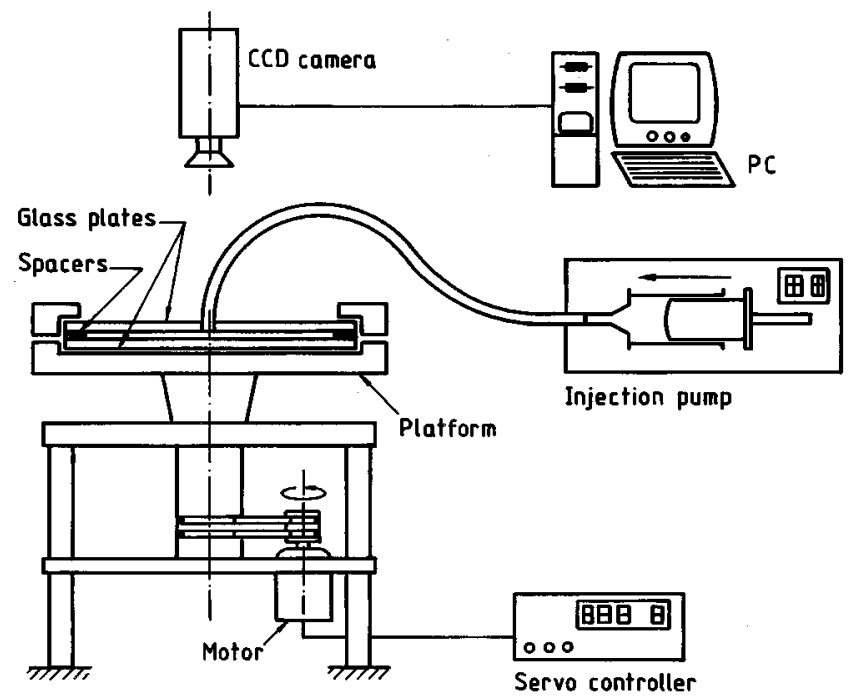

FIG. 2. Scheme of the experimental setup. See the text for details.

$$
n_{c}=\sqrt{1+\frac{\widetilde{\Omega} R^{3}}{\widetilde{\sigma}}}
$$

Note that, contrary to the usual case $(Q>0, \Omega=0)$, for positive $\widetilde{\Omega}$ there is always at least one unstable mode, regardless of how large (small) the surface tension (interface radius) may be.

\section{EXPERIMENTAL SETUP}

The Hele-Shaw cell used in this investigation is made of two circular glass plates, $6 \mathrm{~mm}$ thick and $40 \mathrm{~cm}$ in diameter. The two plates are placed on top of each other, separated by a narrow gap, typically between 0.25 and $1.00 \mathrm{~mm}$. The separation is provided by six calibrated spacers evenly spaced along the periphery of the cell. We estimate the fluctuations in the gap around $0.05 \mathrm{~mm}$. A viscous fluid, Rhodorsil 47 V 50 silicone oil, is injected to the cell by a syringe pump, through a hole drilled in the center of the top plate, using a special connector to prevent twisting of the tube when the cell rotates around its vertical symmetry axis. The syringe pump operates at injection rates adjustable from 1 to $299 \mathrm{ml} / \mathrm{h}$ in steps of $1 \mathrm{ml} / \mathrm{h}$. The oil used has surface tension $\sigma=20.7 \mathrm{mN} / \mathrm{m}$, kinematic viscosity $\nu=50 \mathrm{~mm}^{2} / \mathrm{s}$ at $25^{\circ} \mathrm{C}$, and perfectly wets the glass plates.

The cell is mounted on top of a rotating platform driven by a dc motor and reductor. A scheme of the setup is shown in Fig. 2. The motor incorporates a tachometer, which is used by an external, linear, four-quadrant servocontroller to maintain highly stable angular velocities independently of load fluctuations. Available velocities go from 0.0 to $300.0( \pm 0.1) \mathrm{rpm}$. The axis of the horizontal platform has been machined conical and the setup has been mounted on a heavy granite table with three adjustable feet in order to have the cell accurately leveled even at the highest angular velocities. A proper leveling of the cell is critical during rotation. In addition, the center of the cell is carefully aligned with the rotation axis before each run, to a tolerance less than 0.02 $\mathrm{mm}$ in the radial direction. The illumination is provided by 


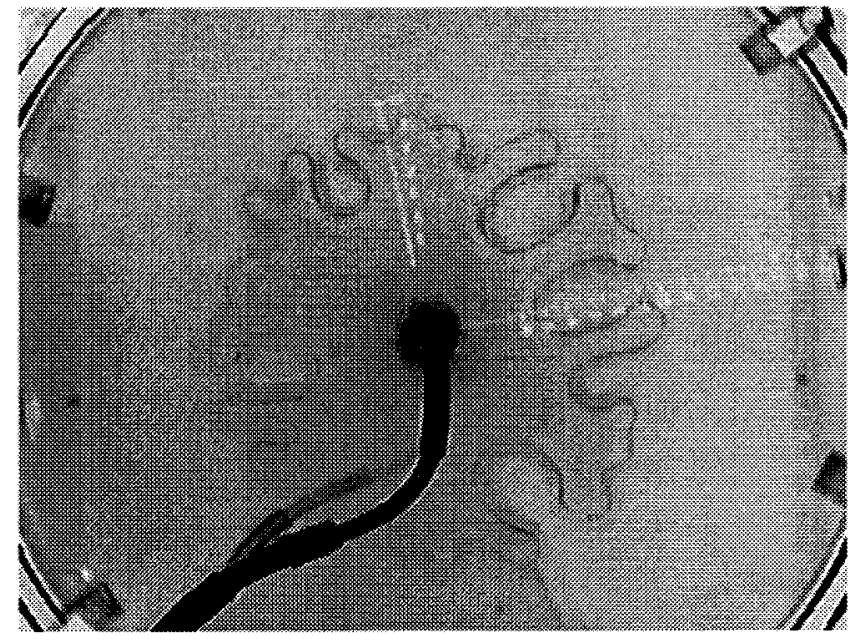

FIG. 3. Typical pattern obtained in our rotating Hele-Shaw cell. Oil is the inner fluid and air the outer one. Here $Q=0, b=0.5 \mathrm{~mm}$, and $\Omega=80 \mathrm{rpm}$. This single picture is extracted from an actual sequence of video frames.

four neon tubes that form a square around the cell, at the level of the gap.

The patterns obtained are recorded with a JVC TK-S340 charge coupled device camera $(512 \times 582$ pixels $)$ equiped with a $8-\mathrm{mm}$ Cosmicar-Pentax objective. The overall illumination is adjusted to make the electronic shutter of the camera operate at its maximum speed in order to avoid blurring the images of the rotating pattern. Images are digitized sequentially by a Vitec VideoMaker frame grabber and stored in the memory of a personal computer. The spatial resolution of the captured images goes from a maximum of $768 \times 576$ pixels for a still image down to $288 \times 384$ pixels for the maximum capture rate, 12.5 images/s, with 256 gray levels per pixel. The number of images in a sequence is limited by the amount of memory in the computer (currently $16 \mathrm{Mb}$ ).

Before each run, the glass plates are thoroughly cleaned and dried to avoid memory effects of wetting films from previous runs. Typically, a stable circular interface is formed first by injecting oil into the cell. Once the oil circle reaches a desired radius $R_{0}$, the injection rate is adjusted to a preselected value and, at the same time, the motor is switched on with the servocontroller adjusted to a preselected velocity $\Omega$. Then the pattern develops and a sequence of images is digitally recorded and stored for further analysis.

\section{RESULTS AND DISCUSSION}

We have carried out experiments for different values of the parameters under experimental control. These have been $b=0.25,0.50$, and $0.81 \mathrm{~mm} ; Q=0,48,69,78,86,111$, 156 , and $222 \mathrm{~mm}^{2} / \mathrm{s} ; \Omega=30,60,75,80,90,100$, and 120 rpm; and $R_{0}=50,65,70,80,90,100$, and $110 \mathrm{~mm}$. Runs with $Q=0$ correspond to the spreading of an initially circular drop upon rotation under conditions of mass conservation. An example of the patterns obtained is shown in Fig. 3.

\section{A. Linear regime}

We focus our attention first on testing the dispersion relation obtained from the linear stability analysis presented in

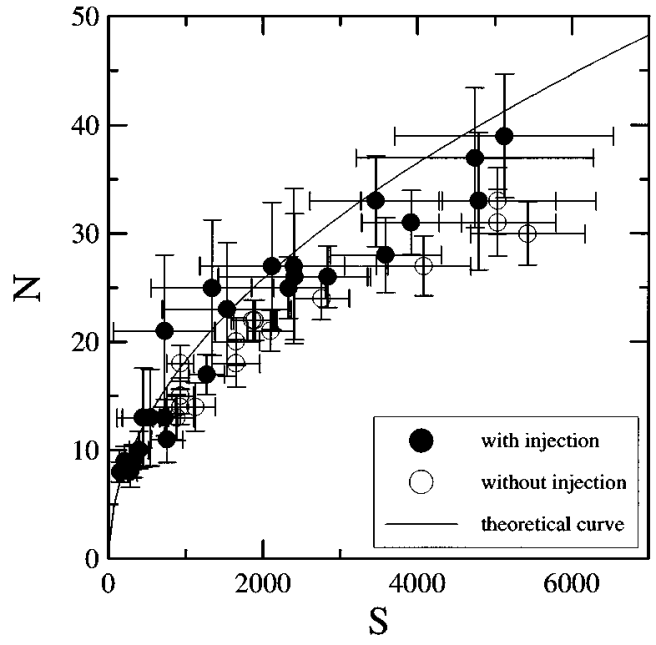

FIG. 4. Number of ripples formed at the onset of instability $N$ as a function of the dimensionless quantity $S=(1 / \sigma)\left(\Omega^{2} R_{0}^{3} \rho\right.$ $\left.-Q R_{0} / 2 \pi M\right)$. Filled circles represent measurements with $Q \neq 0$ and empty circles measurements with $Q=0$ (conserved mass). The solid line is the prediction of a linear stability analysis for $n_{\max }$.

Sec. II. Our experiments correspond to the limit $\rho_{1} \gg \rho_{2}$ and $\mu_{1} \gg \mu_{2}$ since the inner fluid is a silicone oil and the outer one is air. In this limit the expression for the number of fingers developing at a maximum growth rate [Eq. (13)], written in terms of experimental parameters, reads

$$
n_{\max }=\sqrt{\frac{1}{3}(1+S)},
$$

where $S$ is a dimensionless quantity given by

$$
S=\frac{1}{\sigma}\left(\Omega^{2} R_{0}^{3} \rho-\frac{Q R_{0}}{2 \pi M}\right)
$$

and $M=b^{2} / 12 \mu$. We assume that $N$, the number of ripples observed at the onset of instability, is typically of the order of $n_{\max }$. The validity of this assumption relies on two uncontrolled properties of the initial conditions, namely, that the noise is sufficiently weak and that its amplitude does not systematically favor some modes over others. The comparison of the number of ripples and $n_{\max }$ is thus not only a test of the linear dispersion relation but actually of these two assumptions on the external noise in the initial condition. Figure 4 presents the comparison between our prediction Eq. (15) and the results of our observations. The error bars originate from the uncertainties in the cell gap and the original radius $\Delta b=0.05 \mathrm{~mm}$ and $\Delta R_{0}=5 \mathrm{~mm}$. The agreement is remarkable, particularly considering the wideband character of the instability and the lack of control of the noise in the initial condition [12].

We have purposely distinguished between runs with fluid injection and runs without, in which mass is conserved $(Q=0)$. Systematically, patterns with mass conservation exhibit a wave number smaller than predicted by $n_{\max }$ and the discrepancy increases with $S$. In the linear regime, this observation can be understood in the following way. For $Q=0$ the initial drop is unstable for all $\Omega$ (Fig. 1). Since the cell takes a time of the order of $1 \mathrm{~s}$ to reach the steady speed 


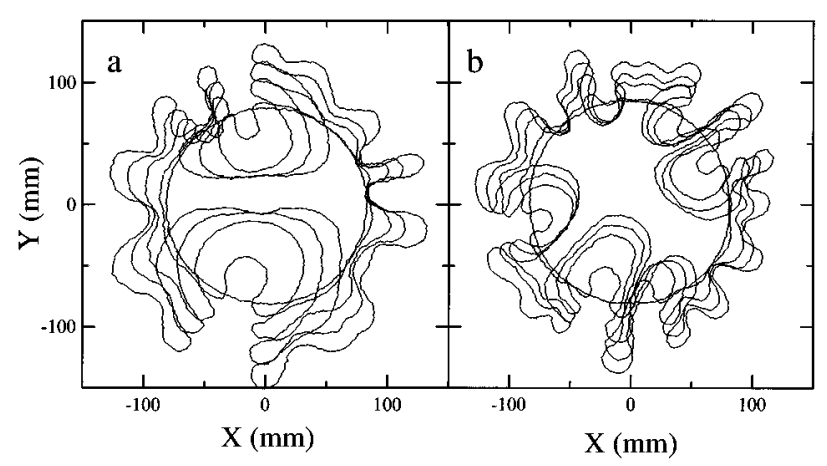

FIG. 5. Two examples of time sequences of the digitized interface. (a) $R_{0}=81 \mathrm{~mm}, b=0.81 \mathrm{~mm}, \Omega=60 \mathrm{rpm}$, and the interface is drawn at $t=0,15.2,24.0,28.8$, and $33.2 \mathrm{~s}$ after the cell started to rotate. (b) $R_{0}=83 \mathrm{~mm}, b=0.5 \mathrm{~mm}, \Omega=60 \mathrm{rpm}$, and $t=0,40.0$, $58.0,66.0$, and $74.0 \mathrm{~s}$. In both runs $Q=0$.

$\Omega$, the interface feels an effective $S(t)$ that grows from zero to its maximum value. By the time $S$ reaches that value, modes with smaller wave number (corresponding to $n_{\max }$ at the instantaneous $\Omega$ ) have been enhanced and may eventually dominate over the most unstable mode at the steady $\Omega$. For $Q>0$ this effect is much less pronounced since the instantaneous $S$ is positive only for a much shorter time.

\section{B. Nonlinear regime with mass conservation $(Q=0)$}

Experiments with mass conservation $(Q=0)$ correspond to the most genuine case to explore with a rotating cell since they correspond to a purely centrifugal driving, with no ana$\log$ in the usual injection-driven setup. In the rest of this section we concentrate on this case as the simplest and most interesting one. The linear regime already shows the particular simplicity of the case $Q=0 . R$ in Eq. (10) is now a constant and the growth and decay of modes are truly exponential. The mode $n=0$ is now marginal and the linear dispersion relation provides us with a natural time scale of the system

$$
\tau=\frac{24 \mu}{b^{2} \Omega^{2} \rho} .
$$

In the linear regime and for $Q=0$ the mobility plays no role other than setting the time scale of the instability and does not affect the emerging pattern, which depends only on the ratio of centrifugal to capillary forces as defined by $S=\Omega^{2} R^{3} \rho / \sigma$ in Eq. (15). Thus $n_{\max }$ is independent of the cell gap and viscosity. Measurements of the number of ripples at the onset of instability, for given $\Omega$ and $R_{0}$ but different $b$ (empty symbols of the same $S$ in Fig. 4), confirm this prediction within experimental uncertainty. However, this is no longer true for well-developed patterns in the nonlinear regime, where substantial differences in morphology indicate that mobility plays an important dynamic role in the nonlinear regime, when fingers develop and compete. An example is shown in Fig. 5, where we compare patterns resulting from two runs for which all parameters have been taken to be the same, except the gap $b$.

In order to study more precisely the resulting patterns and their possible scaling properties we have defined several

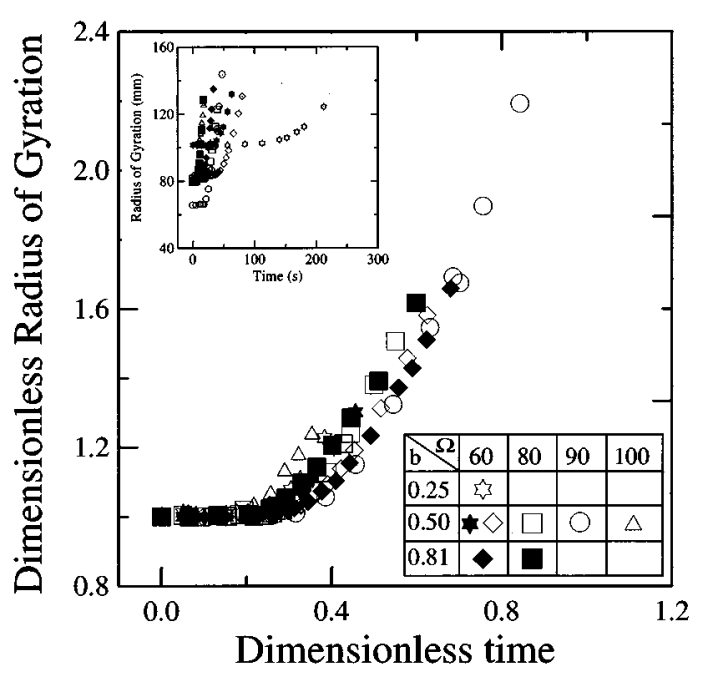

FIG. 6. Collapse of the dimensionless radius of gyration vs dimensionless time, for measurements under different experimental conditions. The inset shows the experimental data before making the variables adimensional. The gap width $b$ is given in millimeters and the angular velocity $\Omega$ in rpm.

quantitative characterizations. Following similar studies in radial Hele-Shaw cells [4], we first study the radius of gyration as the pattern develops in time, as a measure of the mass distribution. The radius of gyration as a function of time is shown in the inset of Fig. 6. This quantity starts from the initial radius $R_{0}$ and rises sharply when the instability develops, at a rate depending on the values of the parameters. We have rescaled time by $\tau$ and the radius of gyration by $R_{0}$ and found that data from runs with completely different sets of parameters collapse into a single curve for all the evolution, as shown in Fig. 6.

We can distinguish three different regimes. First there is a period of latency of about 0.3 in dimensionless time before the onset of instability. Next, a relatively short period with presumably exponential growth follows, and immediately nonlinear effects take over, setting a growth law that appears to be consistent with being linear in time. Remarkably enough, the time of latency itself appears to be a scaling quantity. This is not expected a priori since such time depends on the fluctuations present in the initial condition, which are not controlled in the experiment. In fact, from this characteristic time for the onset of instability we can get an estimate of the actual noise amplitude present in our experiments. In dimensionless time this must be of the order of $\ln \left(\Delta R_{\text {obs }} / \Delta R_{0}\right)$, where $\Delta R_{\text {obs }}$ is the departure from the initial radius of gyration that is first appreciable (Fig. 6 suggests an estimate of 0.05 in dimensionless units) and $\Delta R_{0}$ is the typical relative fluctuation of the initial radius (typical amplitude of modes resulting from preparation of the initial condition). We thus obtain that the noise amplitude in the initial conditions is roughly proportional to $R_{0}$ with an estimate of $\Delta R_{0}$ of $5 \%$ or below.

The second characterization of the emerging patterns is the so-called interface stretching, which measures the increase of the total perimeter of the inner fluid region. Again, the evolution for different experimental conditions is remarkably distinct, as shown in the inset of Fig. 7. The scaling of length and time by $R_{0}$ and $\tau$ in this case does not produce a 


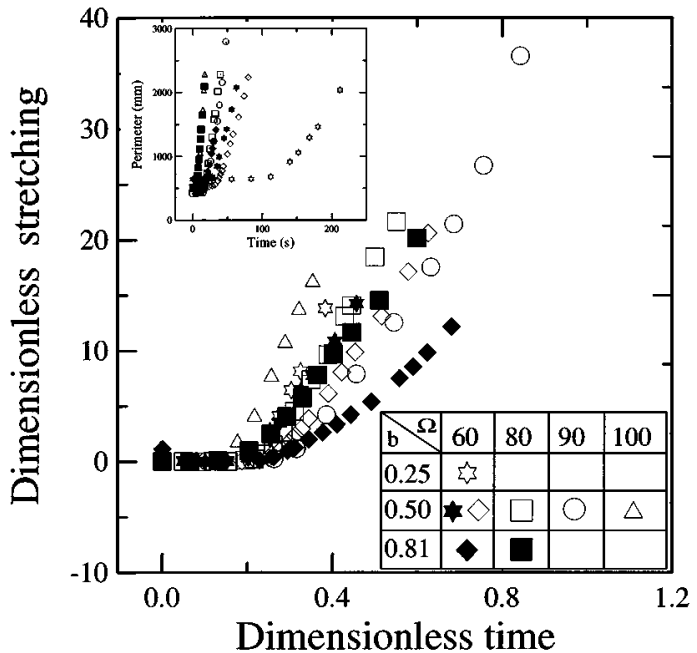

FIG. 7. Dimensionless stretching vs dimensionless time, for measurements under different experimental conditions. The inset shows the experimental data before making the variables adimensional. The gap width $b$ is given in millimeters, and the angular velocity $\Omega$ in rpm.

collapse of the experimental data beyond the latency period. We have not succeeded in finding a reasonably simple scaling that makes our data for the interface stretching collapse in the nonlinear regime. This may be related to the fact that surface tension effects are still significant in the present parameter regime, and there may be more than a single length scale that is necessary to characterize the pattern. The total interface length would presumably be much more sensitive to this fact than other globally averaged quantities such as the radius of gyration.

The reader will notice that the morphologies observed in the rotating cell are apparently quite different from the ones observed in classical radial viscous fingering $(\Omega=0$, $Q>0$ ). Interestingly, a pattern evolution closely resembling ours was obtained by Thomé et al. [13] in the reversed version of the radial viscous fingering experiment $(Q<0$, $\Omega=0$ ) with a viscous fluid being pumped out at the center as air fingers invaded from the exterior. In this high viscosity contrast limit both experiments are quite similar. Our case, however, provides the means to study the competition of such inward fingers with no time limitation, in principle, since the viscous fluid is not being removed from the cell (and could even be injected). In any case, the most interesting differences between the two experiments may be expected at low viscosity contrast.

An interesting way to characterize the competition of inward air fingers, which may be useful to gain some insight into the nonlinear dynamics, is to compare the patterns obtained with the ones in the case of fingers competing in a channel geometry. This can be done by mapping the $(x, y)$ coordinates of the interface into new coordinates $\left[\theta,-\ln \left(r / R_{0}\right)\right]$, where $(r, \theta)$ are the polar plane coordinates. Applying this conformal mapping to the patterns in Fig. 5 produces the result shown in Fig. 8. In this representation, it is apparent that the patterns strongly resemble those found in the classical fingering problem in the channel geometry at high viscosity contrast (with air invading oil). Not only the fingered patterns but the dynamics of competition seem to be

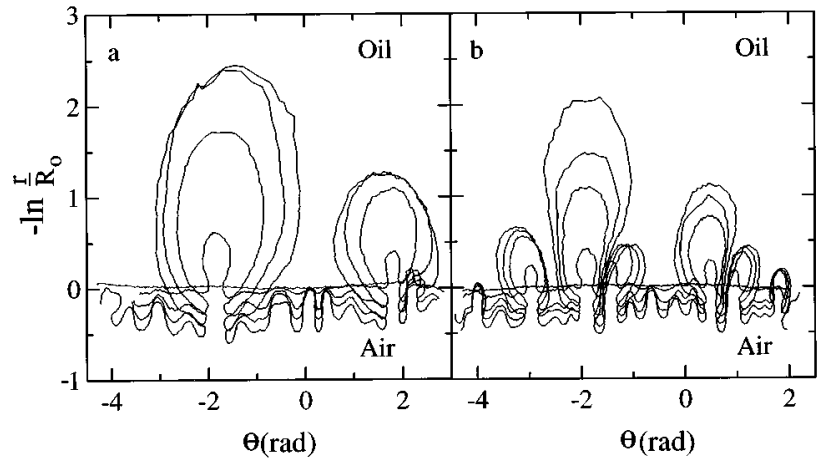

FIG. 8. Time sequences of the same digitized interfaces shown in Fig. 5, mapped here to new coordinates $\left[\theta,-\ln \left(r / R_{0}\right)\right]$. The mapping reveals that air fingers (outer fluid) penetrate the oil (inner fluid). Remember that $Q=0$.

quite similar. In fact, one sees that from a rather noisy initial pattern a competition between fingers is established in which shorter fingers are left behind and only a few of them survive and keep growing. While the pattern in the regions that are left behind is rather static and irregular, the mechanism of finger competition of large fingers seems to follow a rather regular pattern of behavior. This suggests that it is the inward air fingers in our experiments that may exhibit some sort of universal dynamical behavior. With this view, the interface stretching could in fact be too sensitive to the "nonuniversal" regions left out by competition and would then be inadequate for the study of the scaling properties of the patterns.

An important difference with respect to the channel geometry is that the competition process between air fingers seems to stop after a while, when a reduced number of fingers keeps growing (in the logarithmic representation), instead of a single finger, as it would be the case in the experiment in the channel geometry [14]. This observation suggests a scenario in which the competition process is split into two regimes. In the first regime, neighbor fingers are close to parallel to each other and compete essentially like in a channel geometry. After the competition process has eliminated a number of fingers, the angle between them increases to a point at which screening effects no longer produce the dynamical elimination of fingers and a reduced number of them survive, which keep approaching the cell axis.

Within this scenario, the differences between the time sequences of the patterns in Fig. 5 can be easily interpreted. The sequence of interfaces in Fig. 5(a) [and Fig. 8(a)] corresponds to a case in which the number of fingers is small and, consequently, the period of actual finger competition is very short. The sequence in Fig. 5(b) [and Fig. 8(b)] instead would correspond almost entirely to the competition regime between a large number of fingers. With this view, it seems that the larger the number of fingers, the longer the competition regime. A more systematic test of this scenario goes beyond the scope of the present paper.

The observation of the regularity of the behavior of inward fingers lead us to study a third quantitative characterization of the dynamics of fingered patterns in terms of the radial length of selected air fingers as a function of time. We have measured this length for many different runs but dis- 


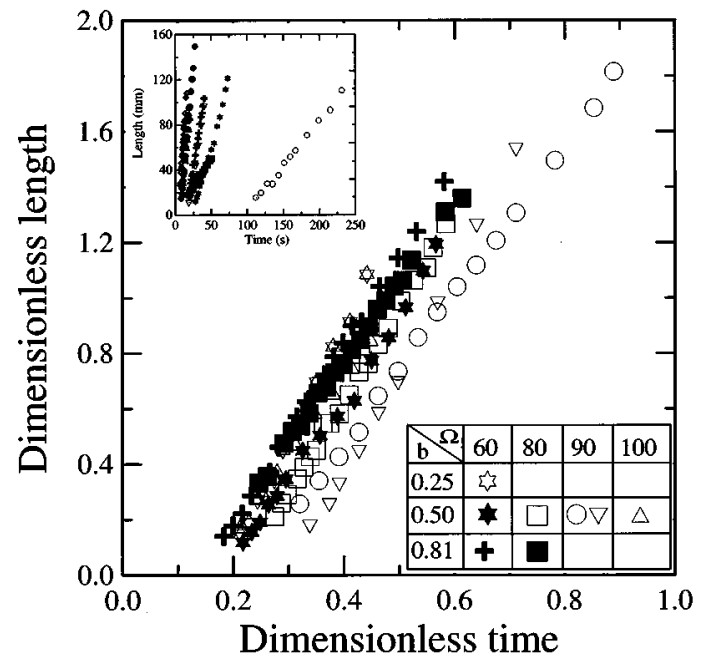

FIG. 9. Collapse of the dimensionless length of individual inward air fingers vs dimensionless time, for measurements under different experimental conditions. The inset shows the experimental data before making the variables adimensional. The gap width $b$ is given in millimeters and the angular velocity $\Omega$ in rpm.

carding fingers that grow preferentially in directions other than the radial direction. The results, presented in the inset of Fig. 9, clearly show that the radial finger growth is basically linear in time for all cases once the finger is formed. When rescaled by $R_{o}$ and $\tau$, the data display the remarkable collapse shown in Fig. 9.

\section{SUMMARY AND CONCLUSIONS}

We have considered a modification of the classical viscous fingering problem in a circular Hele-Shaw cell consisting in a controlled rotation of the cell around its vertical axis. With this modification the instability can be driven by both the density difference and the viscosity contrast between the two fluids. In this sense, our experiments in a rotating cell draw an analogy with gravity-driven experiments in the channel geometry.

The linear stability analysis of our problem has been discussed in detail, showing a rich variety of situations. In the absence of injection, the linear regime is governed by a dimensionless quantity $S$, a balance between centrifugal and capillary effects, and independent of mobility $M=b^{2} / 12 \mu$. The mobility sets in as a relevant dynamic quantity in the nonlinear regime and is then needed to scale the patterns.

An interesting aspect introduced by the presence of centrifugal forcing is that the interfacial instability can be driven without fluid injection $(Q=0)$. Scalings of different magnitudes of the patterns generated deep into the nonlinear regime seem to be more feasible in this case of conserved mass. Attempts of obtaining similar scalings for $Q \neq 0$, in the circular geometry, have had different success in collapsing the experimental data, as discussed in Refs. $[4,15]$.

Previous simulations in the channel geometry showed that magnitudes such as the stretching of the interface, the extent of the mixing zone (equivalent to our finger length), and the root-mean-square displacement from planarity (equivalent to a radius of gyration in the circular geometry) are all equivalent in the sense that mutual linear relations can be estab- lished [16]. This is not the case in our experiments, where a good scaling has been found for the finger length and for the radius of gyration, but not for the stretching of the interface. In all cases, the nonlinear behavior seems to exhibit a linear growth law for the scaled lengths. The failure to scale the interface stretching, however, may be a signal of the existence of an additional characteristic length scale of the patterns. Finally, it has been observed that inward air fingers seem to exhibit a more "universal" dynamical behavior than outward oil fingers in the sense that competition mechanisms can be compared with their counterparts in the channel geometry, through an appropriate conformal mapping. Interestingly, the analogy holds only for an intermediate-time regime, when the number of fingers is quite large. When the pattern has coarsened to a reduced number of penetrating air fingers, the screening effects are eventually affected by the angle of competition and the process stops, leaving a reduced number of active fingers approaching the cell center. Further study of this aspect is necessary to establish the actual validity of this scenario.

Except for the linear regime, the present study has concentrated on the case of conserved mass patterns. Apart from the open questions discussed above for that case, there are many other interesting regimes to be explored. First, we plan to extend our investigation of the limit of high viscosity contrast to the problem with $Q \neq 0$. In particular, it is interesting to look at the different scalings proposed in the literature for $Q>0$ and to check the case $Q<0$, where the instability is driven at the same time by the density difference and the viscosity contrast. A more systematic study of the nonlinear regime, with focus on asympotic behavior, and quantitative analysis of morphologies and symmetries is also of interest. Finally, a slight modification of our setup should make it possible to vary the viscosity contrast and explore in particular the low contrast limit. From what is known concerning the role of viscosity contrast on the mechanisms of finger competition and in the resulting morphologies, the study of this aspect in our problem is expected to be one of its most promising and interesting.

\section{ACKNOWLEDGMENTS}

The authors are indebted to Aurora Hernández-Machado and Jim Maher for their stimulus initiating this research. The technical help provided by Javier Santana, Daniel Binagui, and Josep Lluís Corts, from the Serveis Científico-Tècnics of the University of Barcelona, is gratefully acknowledged. Ll.C. is supported by the Dirección General de Investigación Científica y Técnica (DGICyT, Spain), and F.X.M. by the Direcció General d'Universitats (Generalitat de Catalunya). The work has received the financial support of the DGICyT (Spain) within Project No. PB93-0054-C02-01 and the Comissió Interdepartamental de Recerca i Innovació Tecnològica (Generalitat de Catalunya) within Project No. GRQ1993-01004, and has been developed in the framework of the Training and Mobility of Researchers Programme of the European Community (network Project No. ERB 4061 PL951377).

\section{APPENDIX: CONFORMAL MAPPING FORMULATION}

Here we briefly discuss an alternative formulation of the problem, and a derivation of the linear dispersion relation, 
based on the conformal mapping approach to Laplacian growth problems. This formalism has been applied with great success for high viscosity contrast Hele-Shaw flows (see, for instance, Ref. [1]). We apply it in this same limit to flows in a rotating cell.

The basic idea is to describe the interface dynamics, for either a channel or a circular geometry, in terms of an evolution equation for the conformal mapping $z=f(w, t)$, which maps a prescribed fixed region in the complex plane $\omega$ (in our case the interior of the unit circle) into the physical region corresponding to the viscous fluid, with $z=x+i y, x, y$ being Cartesian coordinates. The evolution equation for the mapping, which contains all the geometric information about the interface, takes the form

$$
\frac{\partial f(\omega, t)}{\partial t}=D f(\omega, t) \widetilde{A}\left[\frac{\operatorname{Re}[D \Phi(\omega, t)]}{|D f(\omega, t)|^{2}}\right],
$$

where $D=\omega \partial_{\omega}$ and $\widetilde{A}[g(s)]$ is an integral operator that acts on a real-valued function $g(s)$, defined on the unit circle $\omega=e^{i s}$, whose output is the complex-valued function that is analytic in the interior of the unit circle and has $g(s)$ as its real part at the unit circle. The complex potential $\Phi(\omega, t)$ in the case of a rotating Hele-Shaw cell reads

$$
\Phi(\omega, t)=\frac{Q}{2 \pi} \ln \omega+\frac{b^{2}}{12 \mu} \sigma \widetilde{A}[\kappa(s)]+\frac{b^{2}}{24 \mu} \rho \Omega^{2} \widetilde{A}\left[|f(s)|^{2}\right],
$$

where $\kappa(s)$ is the curvature of the interface, which is expressed in terms of the mapping as $\kappa(s)=$
$-\operatorname{Im}\left(\partial_{s}^{2} f / \partial_{s} f\left|\partial_{s} f\right|\right)$. We can now derive the linear dispersion analysis for infinitesimal perturbations with integer wave number $n$ of the circular interface, inserting the ansatz

$$
f(\omega, t)=a(t) \omega+\delta(t) \omega^{n+1}
$$

into Eq. (A1). We then obtain

$$
\begin{gathered}
\dot{a}=\frac{1}{a} \frac{Q}{2 \pi}+O\left(\delta^{2}\right), \\
\frac{\dot{\delta}}{\delta}=\omega(n)+O(\delta),
\end{gathered}
$$

where $\omega(n)$ is given by

$$
\omega(n)=-\frac{Q}{2 \pi R^{2}}+\widetilde{\Omega} n-\frac{\widetilde{\sigma}}{R^{3}} n\left(n^{2}-1\right)
$$

and

$$
\widetilde{\Omega}=\frac{\Omega^{2} b^{2}}{12} \frac{\rho}{\mu}-\frac{Q}{2 \pi R^{2}}
$$

and

$$
\widetilde{\sigma}=\frac{b^{2}}{12} \frac{\sigma}{\mu}
$$

[1] D. Bensimon, L.P. Kadanoff, S. Liang, B.I. Shraiman, and C. Tang, Rev. Mod. Phys. 58, 977 (1986).

[2] P. Pelcé, Dynamics of Curved Fronts (Academic, New York, 1988).

[3] D.C. Hong and J.S. Langer, Phys. Rev. Lett. 56, 2032 (1986); R. Combescot, T. Dombre, V. Hakim, Y. Pomeau, and A. Pumir, ibid. 56, 2036 (1986); S. Sarkar and D. Jasnow, Phys. Rev. A 35, 4900 (1987).

[4] S.N. Rauseo, P.D. Barnes, and J.V. Maher, Phys. Rev. A 35, 1245 (1987); S.E. May and J.V. Maher, ibid. 40, 1723 (1989); J.D. Chen, J. Fluid Mech. 201, 223 (1989); S.K. Sarkar and D. Jasnow, Phys. Rev. A 39, 5299 (1989); D. Jasnow and Ch. Yeung, Phys. Rev. E 47, 1087 (1993).

[5] K.V. McCloud and J.V. Maher, Phys. Rep. 260, 139 (1995).

[6] J.V. Maher, Phys. Rev. Lett. 54, 1498 (1985); M.W. DiFrancesco and J.V. Maher, Phys. Rev. A 39, 4709 (1989); and 40, 295 (1989); H. Zhao and J.V. Maher, ibid. 42, 5895 (1990).

[7] G. Tryggvason and H. Aref, J. Fluid Mech. 136, 1 (1983); G. Tryggvason and H. Aref, ibid. 154, 287 (1985); J. Casade- munt, D. Jasnow, and A. Hernández-Machado, Int. J. Mod. Phys. B 6, 1647 (1992).

[8] J. Casademunt and D. Jasnow, Phys. Rev. Lett. 67, 3677 (1991); J. Casademunt and D. Jasnow, Physica D 79, 387 (1994).

[9] P. Constantin, T.F. Dupont, R.E. Goldstein, L.P. Kadanoff, M.J. Shelley, and S.M. Zhou, Phys. Rev. E 47, 4169 (1993).

[10] F. Melo, J.F. Joanny, and S. Fauve, Phys. Rev. Lett. 63, 1958 (1989).

[11] L. Paterson, J. Fluid Mech. 113, 513 (1981).

[12] An equivalent result for the standard viscous fingering problem in circular geometry was previously obtained by Ignés-Mullol and Ortín. The results are reported in Ref. [5].

[13] H. Thomé, M. Rabaud, V. Hakim, and Y. Couder, Phys. Fluids A 1, 224 (1989).

[14] The actual velocity of the fingers at late stages in the channel representation may be distorted by the fact that they are approaching the cell axis in the experiment.

[15] J.D. Chen, J. Fluid Mech. 201, 223 (1989).

[16] D. Jasnow and J. Viñals, Phys. Rev. A 41, 6910 (1990). 